\title{
VIDEOCONFERENCE ACTIVITIES OF THE EURO-ASIAN COOPERATION OF STATE METROLOGICAL INSTITUTIONS UNDER THE PANDEMIC
}

\author{
V. L. Hurevich and N. D. Liakhova
}

UDC 006.91:061.1:004.773.5(4/5)

\begin{abstract}
An overview of the activities of the Euro-Asian Cooperation of State Metrological Institutions (COOMET) concerning the organization of online events using videoconferencing under the COVID-19 pandemic is presented. The choice of an online platform for holding official events under the auspices of COOMET is substantiated. An assessment of the effectiveness of the official events held online is provided. The prospects of using online technology in organizing COOMET activities are outlined.
\end{abstract}

Keywords: metrology, pandemic, coronavirus, videoconference, online format, webinar, national metrology institute, standard, calibration and measurement capabilities.

Introduction. In March 2020, the World Health Organization stated that the massive spread of the COVID-19 had acquired the character of a global pandemic. The pandemic necessitated the reorganization of international cooperation [1], primarily the transition to remote work in various fields. This also affected international cooperation in the field of science and technology, including metrology. Therefore, for the Euro-Asian Cooperation of State Metrological Institutions (COOMET), having the official status of a regional metrological organization (RMO) in the world community and representing the global metrological system [2-4], the introduction of videoconferencing has become a priority task.

The metrological institutions of the Republic of Azerbaijan, the Republic of Armenia, the Republic of Belarus, the Republic of Bulgaria, Bosnia and Herzegovina, the Federal Republic of Germany, Georgia, the Republic of Kazakhstan, the People's Republic of China, the Kyrgyz Republic, the Democratic People's Republic of Korea, the Republic of Cuba, the Republic of Lithuania, Republic of Moldova, Russian Federation, Romania, Slovak Republic, Republic of Tajikistan, Turkish Republic, Republic of Uzbekistan, and Ukraine are collaborating within the framework of COOMET. According to the COOMET Memorandum of Cooperation D1/2020, the objectives of COOMET involve strengthening the relations between state metrological institutions and creating effective mechanisms for solving the following issues:

- achieving mutual compliance of standards and consistency of requirements for measuring instruments and methods of their metrological control;

- recognition of the equivalence of national certificates for the results of metrological activities;

- exchange of information on the state of metrological services and directions of their development;

- joint development of metrological projects (topics); assistance in the mutual provision of metrological services.

Since its establishment, COOMET had carried out its activities in the traditional way: workshops, annual meetings, and other events were held in different countries to discuss topical issues and problems [5, 6]. All this promoted effective cooperation in the areas of COOMET activities stipulated by the Memorandum, including the participation of a number of National Metrology Institutes (NMI) in metrological European scientific programs [7].

Due to the global pandemic, since March 2020, in the COOMET RMO some projects on measurement standard intercomparison for NMI and designated institutes of the COOMET member countries have actually been suspended due to restrictions in terms of border crossing (both for goods and for people). In addition, some planned events were cancelled or

Belarusian State Institute of Metrology (BelGIM), Minsk, Republic of Belarus; e-mail: hurevich@belgim.by, coomet@belgim.by. Translated from Izmeritel'naya Tekhnika, No. 12, pp. 58-63, December, 2020. Original article submitted September 21, 2020. Accepted October 29, 2020. 
postponed. In particular, the 30th meeting of the COOMET Committee (the governing body of the RMO), which was scheduled for April 1-2, 2020 in Nur-Sultan, Republic of Kazakhstan, took place as an online event on September 24, 2020.

Selecting a platform for conducting online events of the RMO. In May 2020, the COOMET Secretariat initiated the topic 811/BY/20 - "Development of the order and procedures for holding official COOMET meetings and events under the auspices of COOMET in the videoconference/webinar format based on the organization and conduct of test videoconferences with the participation of experts from COOMET countries." As part of the topic, a number of test videoconferences/ webinars were held with the participation of members of the created working group and other stakeholders from different countries to examine the advantages of various online communication platforms.

Despite the undoubted advantages of popular services (Skype, Microsoft Teams, Tixeo, Webex, etc.), discussed by the working group, they all have a significant drawback - the impossibility of organizing conference interpreting during online events. In COOMET, two working languages are used (Russian and English); therefore, the possibility of providing interpreting during conferences was decisive when selecting a platform for COOMET online events. Based on the test results, the working group on COOMET topic 811/BY/20 recommended the Zoom service for conducting online COOMET events, since, at present, it is the only platform that provides an opportunity to organize simultaneous interpreting into two languages during online events. Additionally, Zoom provides an opportunity to vote during meetings of COOMET bodies, which is important when organizing official RMO events. In June and July 2020, the Zoom service was tested at four COOMET online events, including two conducted with simultaneous interpreting.

Within the framework of the above-mentioned COOMET topic, short instructions were developed for the participants of videoconferences/webinars. On the basis of these instructions, the "Procedure and practices for holding official meetings of COOMET bodies and events under the auspices of COOMET in the videoconference/webinar format" COOMET Document will be developed.

Hereafter, the information about the most significant COOMET online events is provided.

COOMET events held in online format. International Bureau of Weights and Measures training course (May 25-27, 2020). Under the Capacity Building and Knowledge Transfer Program (CBKT), the International Bureau of Weights and Measures (BIPM) planned to hold a BIPM-KOOMET workshop on the use of the new KCDB 2.0 platform for chairmen of technical committees and technical experts of COOMET on the examination of calibration and measurement capabilities (CMC data) in the traditional format on 22-23 April, 2020 in Minsk. Due to the extraordinary circumstances surrounding the spread of COVID-19, the workshop was held online at the end of May 2020.

The KCDB database is a freely available web resource (KCDB, www.bipm.org/kcdb) related to the implementation of the Agreement on the Mutual Recognition of National Standards and Calibration and Measurement Certificates issued by NMIs (CIPM MRA).

It should be noted that COOMET was the first RMO, with which BIPM organized such an event. The following representatives were appointed as the organizers of the course: Ch. Kuanbaev (BIPM, head of the BIPM CBKT Program), N. Liakhova (COOMET, head of the COOMET secretariat), and Y. Bunyaeva (coordinator of the BIPM CBKT Program from COOMET, National Scientific Center Institute of Metrology, Ukraine). E. Henson - the Director of the International Relations Department of the BIPM, S. Picard - KCDB Coordinator, S. Bergstrand - Executive Secretary of the Joint Committee of the RMI and BIPM (JCRB), S. Manige - Assistant for KCDB, and Ch. Kuanbaev were the speakers at the webinar.

The number of registered participants of the webinar exceeded 100 people from NMIs and designated institutions of the Republic of Belarus, Georgia, the Republic of Kazakhstan, the Republic of Moldova, the Russian Federation, the Republic of Turkey, the Republic of Uzbekistan, and Ukraine. Specialists of some NMIs took part in the webinar both in the format of personal online participation and group viewing of presentations.

Within the framework of the webinar, issues related to the capabilities of KCDB 2.0, the creation of user accounts, the formation and examination of $\mathrm{CMC}$ data, as well as the registration of intercomparison data were considered. The statistics on this database application was supplied. An online survey of the participants was used as a feedback; all the responses received were discussed with the speakers. The participants highly appreciated the organization and relevance of the webinar, approving the activities of COOMET within the BIPM CBKT Program. The results of the online survey and discussion analysis were taken into account when organizing other COOMET online events. 
COOMET expressed gratitude to BIPM for the opportunity to attract a wide range of specialists from NMIs and designated institutes of COOMET member countries and to all speakers for informative reports.

25th meeting of the COOMET President's Council (June 16, 2020). Members of the President's Council, Chairman of the COOMET Joint Committee for Measurement Standards A. Chunovkina, as well as the COOMET representatives of the national secretariats of Germany, the Russian Federation, and Ukraine took part in the meeting.

The following decisions were made by the COOMET President's Council:

- to approve the holding of the 30th meeting of the COOMET Committee by videoconference on the Zoom platform with the participation of COOMET Committee members or their official representatives according to a shortened agenda. On the eve of the 30th meeting of the COOMET Committee, a webinar was to be held for discussing possible directions of joint scientific research;

- to approve a draft of the COOMET Strategy for 2020-2025 and the Roadmap for its implementation; to hold a COOMET webinar for discussing these projects with the participation of COOMET Committee members or their official representatives, members of the working group on the development of COOMET strategy, as well as the chairmen of COOMET structural bodies. The final versions of the draft Strategy and the Roadmap should be submitted for approval at the 30th meeting of the COOMET Committee;

- to approve a draft of the COOMET Development Program for 2020-2022. The final version of the Program shall be submitted for approval at the 30th meeting of the COOMET Committee;

- to approve a draft of the COOMET Training Plan for 2020-2021 and use it as a basis for organizing training events under the auspices of COOMET;

- to hold the 9th International Competition "The best young metrologist of COOMET" in April 2021 (Germany was chosen as a venue on the invitation of COOMET Vice-President P. Ulbig).

Taking into account the uncertain situation with the COVID-19 pandemic, the COOMET President's Council recommended the Zoom platform as a service for holding official COOMET meetings and events under the auspices of COOMET in the videoconference/webinar format in 2020.

In connection with the change in the transitional period for the implementation of ISO/IEC 17025 (extended until 01.06.2021) and taking into account the Resolution JCRB 42/01, the President's Council decided to clarify the document "Policy and Plan of the Technical Committee of the COOMET Quality Forum on the transition to ISO 17034: 2016 and ISO/ IEC 17025: 2017 in assessing the quality management systems of NMI and NI COOMET." A draft of the revised document will be submitted for approval at the 30th meeting of the COOMET Committee. External audits of quality management systems of NMIs of the Russian Federation (Mendeleev VNIIM, VNIIMS, VNIIOFI, VNIIFTRI) and the Republic of Kazakhstan (KazInMetr), which have validity of COOMET certificates on recognition of NMI quality management systems and appointed institutes for compliance with ISO requirements/IEC 17025 and ISO 17034 were previously extended to March 1, 2021, will be held between October 2020 and February 2021.

Due to the shift in the schedule of meetings of COOMET governing bodies, caused by the COVID-19 pandemic, the 31st meeting of the COOMET Committee will be held in June 2021. Considering that the 30th anniversary of COOMET foundation will be celebrated on June 12,2021, the meeting of the Committee on these dates will make it possible to solemnly celebrate this event. The place and dates will be agreed as the work proceeds.

The 26th meeting of the COOMET President's Council is scheduled for the second half of February 2021 (this event is planned to be held in Germany or via videoconference, depending on the current situation with the pandemic).

18th meeting of the Joint Committee for Measurement Standards (July 9, 2020). The meeting of the Joint Committee for Measurement Standards (JCMS) was attended by the chairmen of the technical committees, members of the JCMS, representatives of the COOMET Secretariat, and the secretariats of the aforementioned technical committees. The meeting was chaired by the Chairman of the JCMS A. Chunovkina (Mendeleev VNIIM).

During the meeting, the following issues were considered:

- implementation of projects initiated by the JCMS (topic 794/RU/19 "Translation and adaptation of the new versions of CIPM MRA documents," as well as the "Recommendations of the Working Group on the implementation and 
application of the CIPM MRA" document and topic 812/RU/20 "Development of COOMET Recommendations on ensuring traceability of measurement results/standards in COOMET");

- updating and implementation of the COOMET Program of Intercomparisons in 2020;

- decisions of the COOMET Committee made based on the results of electronic voting: amendment of the Memorandum of COOMET Cooperation and appointment/extension of the powers of the heads of a number of technical committees and subcommittees; requirements of the Document COOMET D5: 2020 "Model Regulations on the structural body of COOMET;" the need to update the Regulations on the JCMS and its technical committees, as well as the appointment/ extension of the powers of the heads of a number of technical committees and subcommittees;

- approval of the new composition of the COOMET Board of Appeal on the issues of intercomparisons of national measurement standards and examination of $\mathrm{CMC}$ data;

- discussion of the progress of work on the development of the COOMET Roadmap for the implementation of decisions related to the redefinition of the basic units of the International System of Units (SI);

- results of the CMC line publication in 2019-2020 and participation in the examination of COOMET and other RMO CMC lines; the specifics of the expertise organization during the transition to KCDB 2.0;

- updating the composition of the COOMET technical expert groups for the examination of CMC data on the types of measurements that fall within the responsibility of COOMET technical committees;

- the results of the BIPM-COOMET webinar (a short online course on KCDB 2.0, May 25-27, 2020) and further actions of the JCMS technical committees in the field of training COOMET technical experts on the examination of CMC data;

- posting information at www.coomet.net (on the webpages of the JCMS and its technical committees) and for online editors of the pages of technical committees;

- checking the scientific and technical level of COOMET publications in the thematic areas of cooperation assigned to structural bodies;

- discussion of the draft informational material of COOMET on conducting distance intercomparisons and calibrations of COOMET NMI standards;

- discussion and submission for approval of COOMET publications updated in 2019-2020 (COOMET Recommendation R/GM/15 "Procedure for issuing calibration certificates of national metrology institutes within the CIPM MRA" and COOMET Recommendation R/RM/22 "Form and content of COOMET certificate for reference materials of composition and properties of substances and materials");

- organization of meetings for technical committees in 2020, including the specifics of their online holding.

The adopted decisions have been discussed at the meetings of the JCMS technical committees and will be submitted for approval by the COOMET Committee.

COOMET webinar on discussing a draft of the COOMET Strategy for 2020-2025 and the 5th meeting of the working group on the COOMET development strategy (July 13, 2020). At the first COOMET webinar, a draft of the COOMET Strategy for 2020-2025, the Roadmap for its implementation, as well as the institutional status of COOMET were discussed. The webinar organized by the COOMET Secretariat was attended by 38 specialists from COOMET member countries, including COOMET Vice-Presidents, COOMET Committee members or their representatives, Chairmen of COOMET Technical Committees, representatives of COOMET Secretariat, and national secretariats of COOMET RMO member countries.

COOMET Vice-President P. Ulbig reported on the results of the "Status and Prospects of COOMET" external assessment, which was carried out in 2018-2019 by independent experts and included personal interviews and online surveys of cooperation participants. The results of the external evaluation were previously discussed within the framework of the working group on the development of the COOMET strategy. As a result of the working group activity, the mission and vision of COOMET, as well as seven strategic objectives of COOMET were formulated:

- promoting the international recognition of national measurement standards;

- promoting the recognition of the quality management systems of NMIs and designated institutes of the COOMET member countries;

- providing support to COOMET member countries in obtaining the status of membership in the Metric Convention; - assistance in the implementation of international practice in legal metrology; 
- promotion of professional development and knowledge transfer in COOMET member countries;

- organization of innovative scientific research in the field of metrology;

- internal development of COOMET and external representation.

In order to solve the above tasks, at the beginning of 2020, a draft of the COOMET Strategy for 2020-2025, the Roadmap for its implementation, and the COOMET Development Program for 2020-2022 were prepared after preliminary revision by COOMET Committee members and chairmen of its structural bodies.

At the webinar, the presentation made by the COOMET President about the developed draft documents was discussed. The webinar participants approved the prepared projects in general and recommended to submit them for official approval to the COOMET Committee.

The second part of the webinar was devoted to discussing the feasibility of changing the institutional status of COOMET and its possible forms in the future. COOMET Vice-Presidents P. Ulbig, S. Golubev, and P. Neyezhmakov presented reviews on the status and procedure of funding in other regional organizations (EURAMET, WELMEC, AFRIMETS, APMP, and SIM). It was noted that EURAMET, WELMEC, and SIM had already been registered as legal entities and that AFRIMETS and APMP continued discussions about this question.

The COOMET President substantiated the need to update the fundamental COOMET documents defining COOMET membership and the status of the organization. During the exchange of views, the representatives of the partner countries confirmed that the documents should be corrected in order to involve all NMIs and designated institutes of the COOMET member countries in active cooperation within COOMET. It was agreed that the discussion of the entire block of issues on COOMET reforming will be continued within the framework of the strategy working group (taking into account the opinions expressed at the webinar) and submitted for discussion by the COOMET Committee.

At the end of the webinar, the 5th meeting of the working group on strategy was held, where all members came to an agreed opinion on the need to intensify the work on assessing the possibility of establishing COOMET as a legal entity. To this end, it was decided to carry out a survey among the members of the COOMET Committee followed by examination of the results at the next meeting of the COOMET Committee.

30th COOMET Committee online meeting (September 24, 2020). The event was attended by COOMET Committee members or their official representatives from 17 countries (Republic of Azerbaijan, Republic of Armenia, Republic of Belarus, Bosnia and Herzegovina, Federal Republic of Germany, Georgia, Republic of Kazakhstan, People's Republic of China, Kyrgyz Republic, Lithuanian Republic, Republic of Moldova, Russian Federation, Slovak Republic, Republic of Tajikistan, Turkish Republic, Republic of Uzbekistan, and Ukraine).

On the basis of the discussions around measures aimed at improving COOMET activities, the following decisions and actions were taken:

- mission and vision of COOMET were approved;

- the COOMET Strategy for 2020-2025 and the Roadmap for its implementation were approved;

- the COOMET Development Program for 2020-2022 (P1/2020) was approved, etc.

The COOMET Committee members decided to extend the powers of the current COOMET President V. Gurevich (Belarus) for the period from April 2021 to April 2024 (on the basis of Clause 2, Section 5 of the COOMET Memorandum of Cooperation).

At the meeting, the preliminary results of a questionnaire survey of the COOMET Committee members on the selection of countries, where a legal entity can be registered for the needs of COOMET, were discussed. Further, the COOMET strategy working group will discuss the selection of potential countries of the legal entity location for the needs of COOMET and assess the legal and organizational issues related to the registration of a legal entity for the selected countries (with the involvement of lawyers), as well as discuss further actions for a possible solution of the problem 1.2.3 "Formation of proposals on the organizational and legal form of COOMET; including funding mechanisms." In order to discuss the results obtained at the COOMET Committee meeting in 2022 and to decide on the advisability of changing the institutional status of COOMET, the preparation of the final report and the Roadmap with proposals for the implementation of the tasks of the working group on strategy will be completed by October 2021. 
The COOMET Committee approved the COOMET Roadmap for the implementation of decisions related to the redefinition of the basic units of the International System of Units (SI).

During the meeting of the COOMET Committee, the issues of implementation of the CIPM MRA Agreement were considered (in terms of the examination of CMC data, intercomparisons of national standards, assessment of quality management systems of NMIs and designated institutes of COOMET member countries, etc.).

The COOMET Committee instructed the Chairmen of JCMS and TC 1.2- TC 1.11 to analyze the state of work on the COOMET intercomparisons lasting more than 5 years. Information on this issue and the proposed COOMET corrective actions in February 2021 will be sent to JCRB.

The COOMET Committee requested all its members and heads of NMIs and designated institutes of COOMET member countries to provide maximum assistance in carrying out international comparisons of measurement standards and to facilitate the completion of COOMET manned comparisons.

The meeting approved the revised "Policy and plan of TC 3.1"Technical committee of the quality Forum" document for the transition to ISO 17034: 2016 and ISO/IEC 17025: 2017 in assessing the quality management systems of NMIs and appointed COOMET institutes" (taking into account the JCRB decision to change the transition period for the implementation of ISO/IEC 17025: 2017). Additionally, the schedule of external audits of quality management systems of NMIs and designated COOMET institutes in 2020-2021 was discussed.

During the meeting of the COOMET Committee, the issues of cooperation in the field of legal metrology, information, and training of metrologists were also considered. The meeting participants were provided with information on the current state of the work program of TC 2 "Legal metrology" for 2019-2020 and TC 4 "Information and training" for 2018-2020.

COOMET Committee approved updated COOMET publications:

- COOMET Recommendation R/GM/15: 2020 "Procedure for issuing calibration certificates of national metrological institutes and designated institutes under the CIPM MRA;"

- COOMET Recommendation R/RM/22: 2020 "Form and content of COOMET certificate for reference materials of composition and properties of substances and materials;"

- COOMET P5/2020 Program for Joint Development of Reference Materials within COOMET;

- Informational material COOMET I/RM/2: 2020 "Register of reference materials of composition and properties of substances and materials developed within COOMET."

The next meeting of the COOMET Committee will take place on June 15-17, 2021 together with the meeting of the JCMS and the "COOMET - 30 years" workshop.

In addition to the events listed above, by the end of 2020, online meetings of almost all structural bodies of COOMET were held, including the COOMET webinar aimed at discussing possible directions for joint scientific research.

Conclusion. The practice of introducing videoconferencing into COOMET activities became a forced step in the context of the pandemic, yet proved to be effective in ensuring continuous work of COOMET RMOs during the period difficult for the entire world community. COOMET timely selected the Zoom platform as a service for holding online official events under the auspices of COOMET. The conducted analysis of the results of the past COOMET online events proved their high efficiency. The use of online technologies in COOMET activities has good prospects. Among the advantages is the possibility of organizing events in a timely manner and attracting a wide range of specialists from different countries (which is not always possible when organizing meetings and training events in the offline format).

In the future, when holding RMO events, a combined approach can be used: some participants will be present in person, while others having no opportunity or sufficient funding for face-to-face visits will be able to join their colleagues using online technologies.

\section{REFERENCES}

1. S. M. Markedonov, "Pandemic Lessons for International Relations," Mezhdunar. Analit., 11, No. 1, 7-9 (2020).

2. $\quad$ E. Zaydel, “Towards a global metrological system,” Zakonodat. Prikl. Metrol., No. 1, 2-4 (2004). 
3. L. K. Isaev, "From national measurement systems to the World measurement system," Zakonodat. Prikl. Metrol., No. 6, 2 (2019).

4. "Updated joint BIPM, OIML, ILAC, and ISO Declaration on metrological traceability," Metrol. Priborostr., No. 1, 54-56 (2019).

5. V. I. Belotserkovskii and S.V. Korostin, "International cooperation in the field of metrology," Kompetentnost, No. 5-6 (34-35), 58-62 (2006).

6. V. L. Hurevich, "COOMET. The focus is on the quality of measurements," Mir Izmer., No. 3, 14-20 (2019).

7. B. P. Gorshkov, "Towards the set goals. Metrology for new technologies (review of projects of European metrological programs)," Zakonodat. Prikl. Metrol., No. 1 (146), 34-40 (2017). 